\title{
RESEARCH ON MARINE PHOTOVOLTAIC POWER FORECASTING BASED ON WAVELET TRANSFORM AND ECHO STATE NETWORK
}

\author{
Du Xinhui, Ph. D., \\ Wang Shuai, M.Sc. \\ Zhang Juan,M.Sc., \\ College of Electrical and Power Engineering, Taiyuan University of Technology, Taiyuan, Shanxi, China
}

\begin{abstract}
With the rapid development of photovoltaic power generation technology, photovoltaic power generation system has gradually become an important component of the integrated energy system of marine. High precision short-term photovoltaic power generation forecasting is becoming one of the key technologies in ship energy saving and ship energy efficiency improving. Aiming at the characteristics of marine photovoltaic power generation system, we designed a highprecision power forecasting model (WT+ESN) for marine photovoltaic power generation system with anti-marine environmental interference. In this model, the information mining of the photovoltaic system in marine environment is carried out based on wavelet theory, then the forecasting model basing on echo state network is construct ed. Lastly, three kinds of error metrics are compared with the three traditional models by Matlab, the result shows that the model has high forecasting accuracy and strong robustness to marine environmental factors, which is of great significance to save fuel for ships, improve the energy utilization rate and assist the power dispatching and fuel dispatching of the marine power generation system.
\end{abstract}

Keywords: Marine Photovoltaic Power System; Power Forecasting; Wavelet Transform; Echo State Network

\section{INTRODUCTION}

For large-scale ocean-going ships, the amount of light during navigation is generally adequate (non-rainy days) [1,2], which performs well in ship stability and wind resistance due to larger upper space and deck area. So it is feasible to install a considerable number of photovoltaic panels and other related equipment, at present, the photovoltaic system based on a variety of ship types has also been put into long-term experimental research or commercial operations. Large and medium-sized ships usually have independent power stations, the photovoltaic power generation system can be incorporated into the ship's power grid through the inverter $[3,4,5]$, thus reducing the power generation load of the power station. Due to the limited supply of fuel in the marine environment [6,7], and the influencing factors such as radiation and temperature fluctuate $[8,9]$, it's very important to forecast the power of the photovoltaic power generation system accurately, thus helping to save fuel, improve energy efficiency, and assist the marine power generation system of power scheduling and fuel dispatch.

In order to forecast the ship's photovoltaic power generation accurately, we can start from two aspects: 1). Carry out more accurate data preprocessing. Because the photovoltaic power generation system in the marine environment is susceptible to complex factors, the power sequence often produces more noise. The principle of Wavelet Transform (WT) $[10,11]$ is to reduce the noise in the information by using wavelet multiresolution [12]. And then extract the regular components in the low frequency band in order to separate the stationary signal and the random signal, so it is suitable for the data processing and information mining of the marine photovoltaic power generation system; 2). Build a more accurate neural network. Echo State Network [13,14,15] (ESN) first proposed 
by Professor Jaeger of Germany in 2001 .The main feature of the ESN is that the dynamic reserve pool composed of a large number of neurons randomly generated and sparsely associated with each other is regarded as the hidden layer of the network. Compared with the traditional neural network, ESN has more neurons, and neurons connected randomly, once generated will remain unchanged [16].The network effectively avoids the problem that the network structure is difficult to be determined and the burden of computing is too heavy [17]. It has outstanding performance in modeling complexity and convergence speed, algorithm stability and global optimality and it is well suited for establishing time series models and forecasting.

Based on the WT theory and ESN, a high-precision power forecast model (WT+ESN) [18] for marine photovoltaic power generation system was designed. The experiment was carried out using $15 \mathrm{~kW}$ ship PV monitoring data from ORNL laboratory in USA. The WT+ESN model was compared with the three traditional models by using the three error indexes RE, MAPE and RMSE. It is found that the accuracy of WT+ESN model is the highest, and the forecast network is more robust to the marine environmental factors. The overall performance is the best, and it is very suitable for the power forecast of the marine photovoltaic power generation system. It is of great significance to improve the energy efficiency of the marine power generation system.

\section{EXPERIMENTAL}

\section{INFORMATION MINING OF PHOTOVOLTAIC POWER SEQUENCES BY WT}

The influencing factors such as the amount of radiation, temperature and other factors in the marine environment will affect the marine photovoltaic power generation system, so that there will be a variety of complex information into the power sequence. And the regularity component is generally in the low frequency band of the original signal, the random component is generally in the high frequency band. In order to reduce the influence of noise on forecasting accuracy, the original signal was decomposed by wavelet decomposition technique, and the corresponding sub-sequence was de-noised by reconstruction technique.

We use the PV monitoring data from the US ORNL laboratory, collected in 2015 the annual solar radiation, ambient temperature and 15kW ship photovoltaic power generation system output power. The effective data of August and September were selected and pretreated by wavelet transform. As the photovoltaic panels usually work in the daily 7: 00-18: 00 power output, the remaining time the power output is basically zero, so sampling 432 points for the forecast analysis. Photovoltaic original output sequence is shown in Fig. 1.

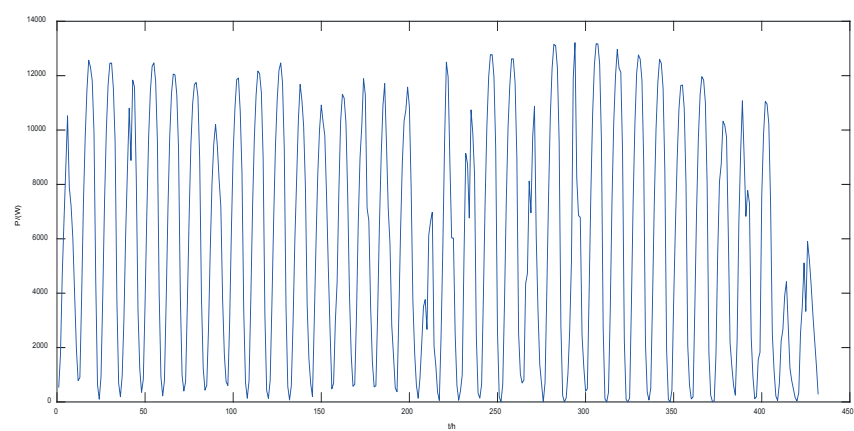

Fig. 1. Photovoltaic raw output sequence diagrams

The original power sequence $\mathrm{P}$ is decomposed by 3 -scale wavelet and single reconstruction. After decomposition of the original power sequence, the regular components al, $\mathrm{a} 2, \mathrm{a} 3$ and the detail components $\mathrm{d} 1, \mathrm{~d} 2, \mathrm{~d} 3$ were obtained. To effectively retain the useful signal in the high frequency signal, the $\mathrm{d} 1$ and $\mathrm{d} 2$ sequence containing more noise were subjected to secondary wavelet transform and noise reduction processing, and the high frequency part of the detail component was ignored. The residual components were reconstructed by $\mathrm{WT}$, and the $\mathrm{d} 1$ and $\mathrm{d} 2$ sequences were de-noised. The results are shown in Fig. 2 and 3.

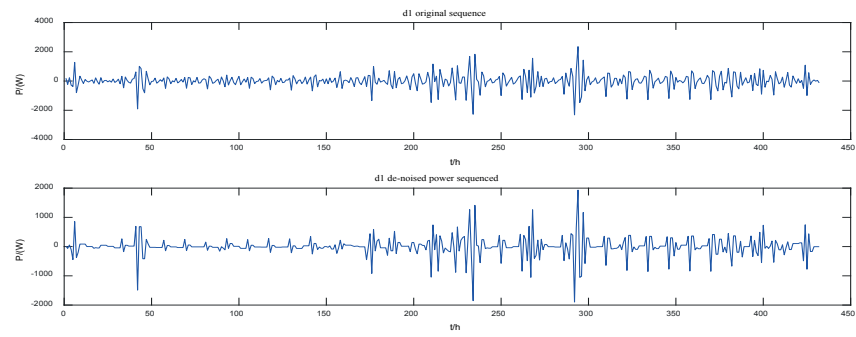

Fig. 2. d1 sequence wavelet de-noising contrast
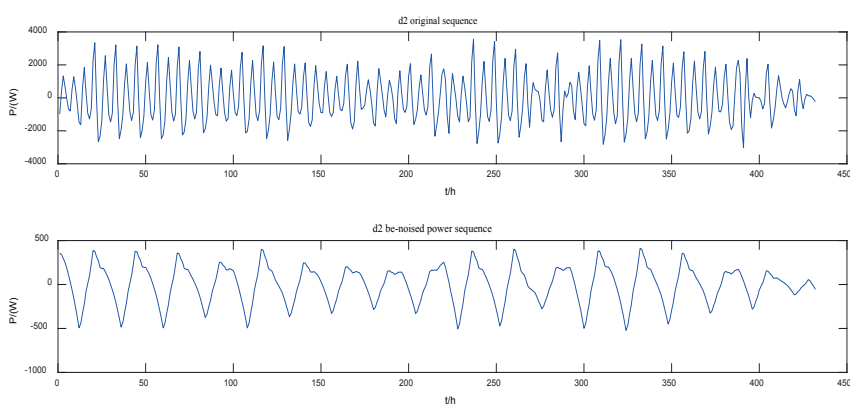

Fig. 3. d2 sequence wavelet de-noising contrast

From the results of Fig. 2 and 3, it can be seen that the amplitude and frequency of $\mathrm{d} 1$ and $\mathrm{d} 2$ are effectively reduced after wavelet de-noising, and the effect of $\mathrm{d} 1$ is more obvious, which is in accordance with the characteristics of wavelet analysis. Fig. 4 shows the relationship between the de-noising of the photovoltaic original power sequence before and after the solar radiation, and it can be seen that the power is 
de-noised and the radiation is closer to the linear relationship and the data is more concentrated.

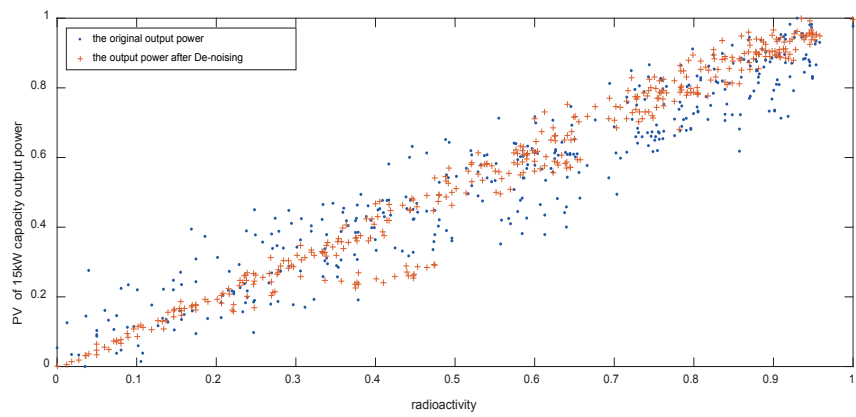

Fig. 4. Original power de noising before and after radiation diagram with the sun

\section{CONSTRUCTION OF ESN}

ESN is a new type of recurrent neural network, which simplifies the network design and training process. ESN structure is shown in Fig. 5 , the overall structure consists of an input layer, a reserve pool, and an output layer [19]. There is 1 node in the input layer, $\mathrm{n}$ nodes in the reserve pool, and $\mathrm{m}$ nodes in the output layer. The solid link weight in the figure is required for the ESN, and the dotted part of the connection weight is selected depending on the situation. Corresponding $t$, the input vector $u(t)$, the internal state vector $x(t)$ and the output vector $y(t)$ of the network are expressed as Eq. (1):

$$
\left\{\begin{array}{l}
u(t)=\left[u_{1}(t), u_{2}(t), \ldots, u_{l}(t)\right]^{T} \\
x(t)=\left[x_{1}(t), x_{2}(t), \ldots, x_{n}(t)\right]^{T} \\
y(t)=\left[y_{1}(t), y_{2}(t), \ldots, y_{m}(n)\right]^{T}
\end{array}\right\}
$$

ESN reserve pool neuron update equation is as Eq. (2):

$$
x(t+1)=f\left(W^{i n} u(t+1)+W x(t)+W^{\text {back }} y(t)\right)
$$

In Eq. (2):

$x(\mathrm{t})$ - The value of the internal state vector at time $t$;

$W^{\text {in }}$ - The input weight of the input signal to the reserve pool;

$W$ - The internal pool of the reserve pool weight;

$W^{\text {back }}$ - The feedback weight of the output layer to the reserve pool.

The output equation of ESN is as Eq. (3):

$$
y(t+1)=f^{\text {out }}\left(W^{\text {out }}(u(t+1), x(t+1), y(t+1))\right.
$$

In Eq. (3):

$W^{\text {out }}$ - The connection weight between the input layer, the reserve pool and the output layer to the output layer;

fout - The excitation function of the output layer, which usually takes the constant value.

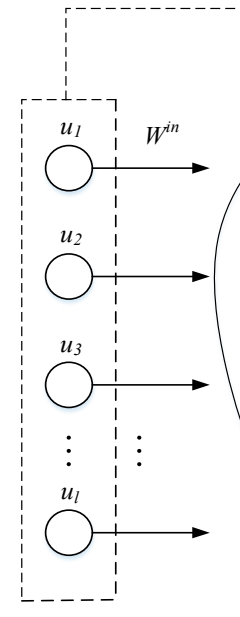

Input layer

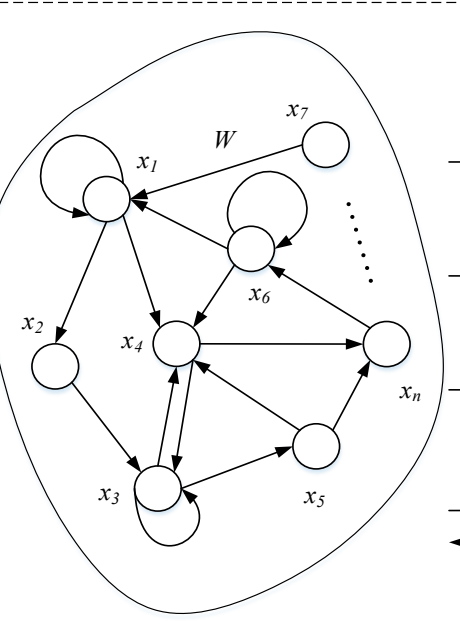

reserve pool

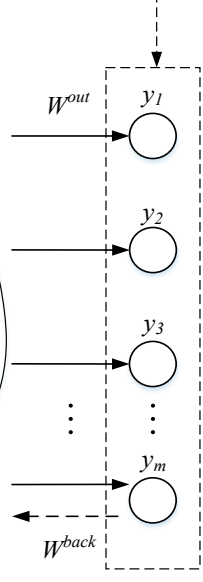

output layer
Fig. 5. The structure of ESN

ESN training is a series of processes based on the training sample set $\left(u(t), y^{*}(t), t=1,2, \ldots, s\right)$ to get the network output weight matrix $W^{\text {out }}$. ESN training process is divided into two stages: sampling stage and weight calculation stage [20]. ESN network training process is shown in Fig. 6.

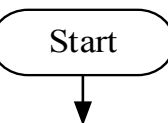

Initialized ESN network parameters, reserve pool size $n$, the excitation function $f$, sparse degree $(S D)$, the spectral radius $(S R)$, and input unit scale $(S C)$, randomly generate the connection weight matrix, $\mathrm{x}(0)=0$.

The sample data is input to the network, the internal state vector $x$ and the output vector $y$ are calculated to update the pool state.

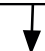

From a certain moment, the internal state vector $x$, the sample input $u$ and the sample output $y^{*}$ are recorded, the matrix $B$ and $T$ are constructed.

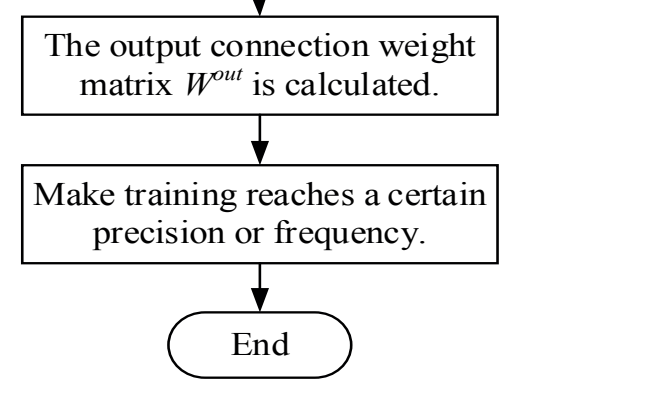

Fig. 6. ESN network training flow chart 


\section{WT+ESN FORECAST MODEL}

Based on WT theory and ESN, a power forecast model (WT+ESN) for high precision marine photovoltaic power generation system with anti-marine environmental interference is designed.

As shown in Fig.7, the WT+ESN forecast model is divided into two steps, wavelet decomposition and single reconstruction of the PV sequence and ESN forecast and linear integration. The whole idea is to preprocess the PV output power using WT to generate sub-sequences of different scale features, which are forecasted by ESN model respectively. Then the PV output sub-sequence is linearly superimposed to obtain the photovoltaic output forecast value.

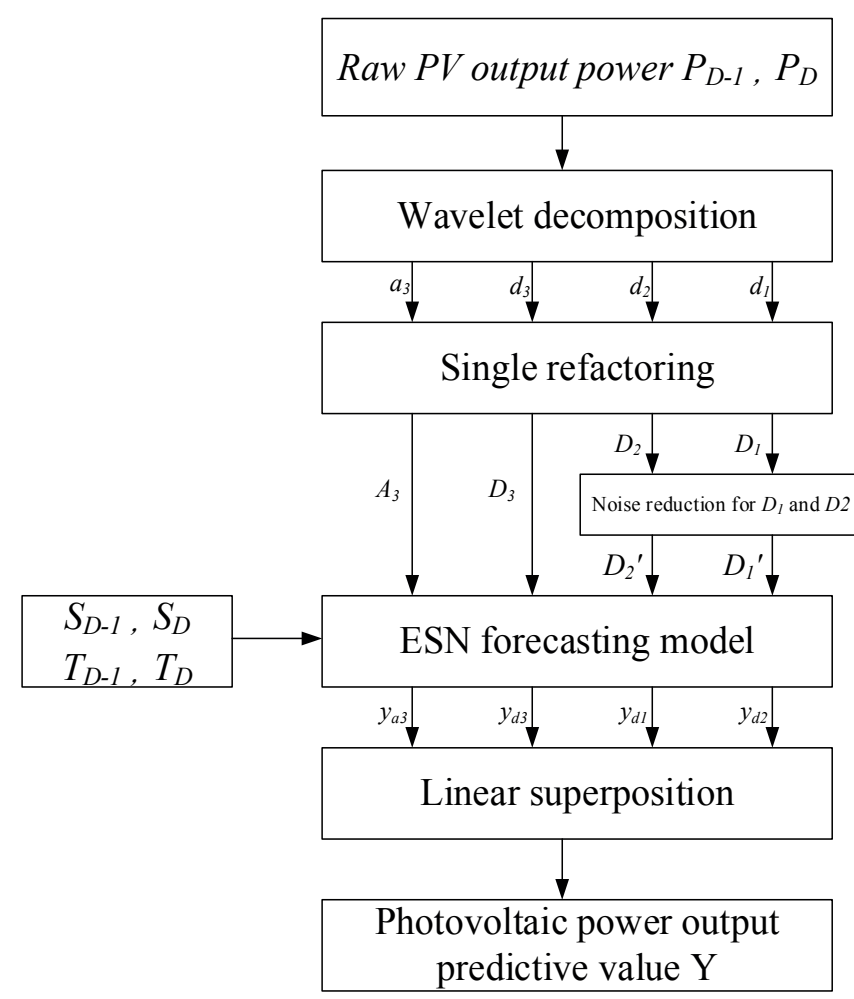

Fig. 7. WT + ESN model to predict flow chart

\section{RESULTS AND DISCUSSION}

For the photovoltaic power generation characteristics, and ESN parameters of the selected principles, the parameters of the ESN network are finally set, the size of the ESN reserve pool is 400 , the sparse degree is $5 \%$, the spectral radius is 0.1 , the input scale and the displacement are 0.6 and 0 respectively.

In order to evaluate the performance of the model, the ESN forecast model, the BP neural network model, the WT+ESN model and the $\mathrm{WT}+\mathrm{BP}$ model were established respectively, and the selected samples were analyzed by wavelet de-noising and no de-noising. Simulation results Fig. 8 and 9 shows the PV power forecast in the sunny day, and Fig. 10 and 11 show the PV power forecast in the cloudy day.

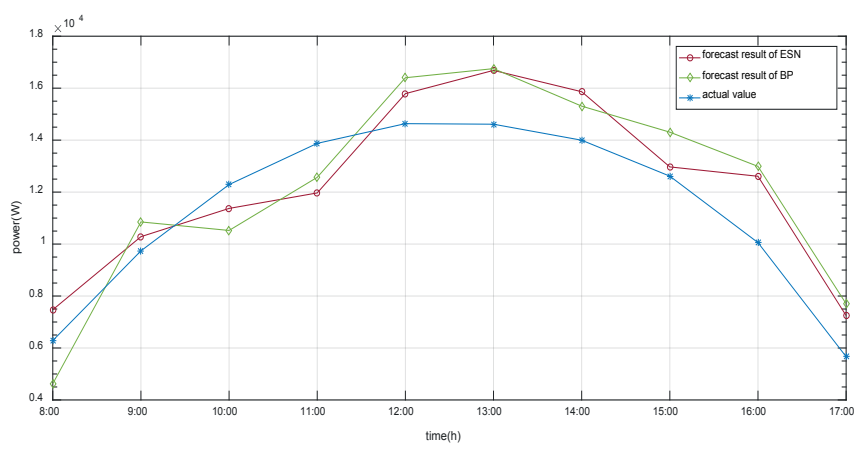

Fig. 8. BP with the ESN model forecasted results (sunny)

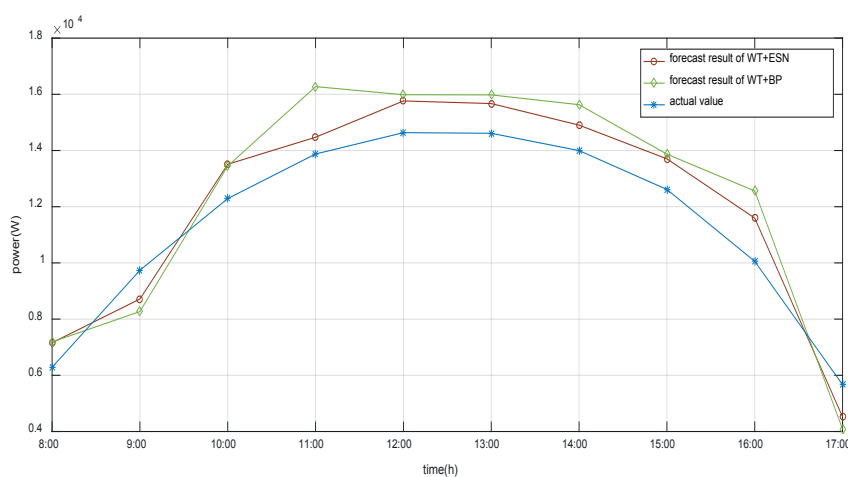

Fig. 9. $W T+B P$ and $W T+E S N$ model forecasted results (sunny)

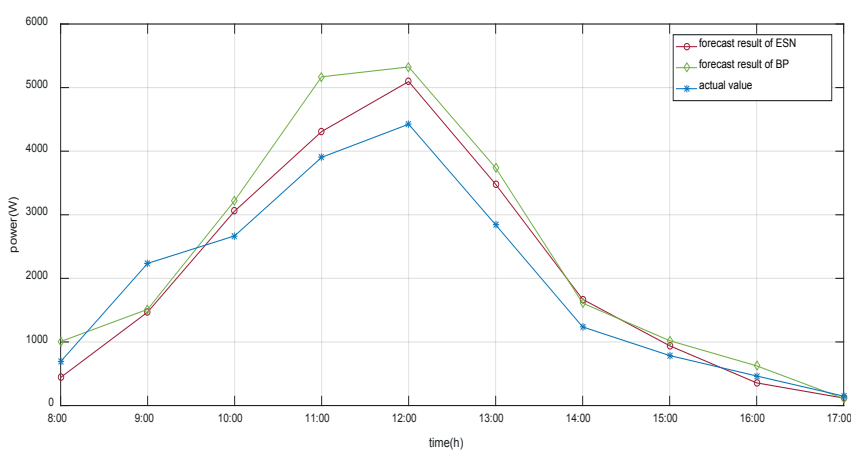

Fig. 10. BP with the ESN model forecasted results (cloudy)

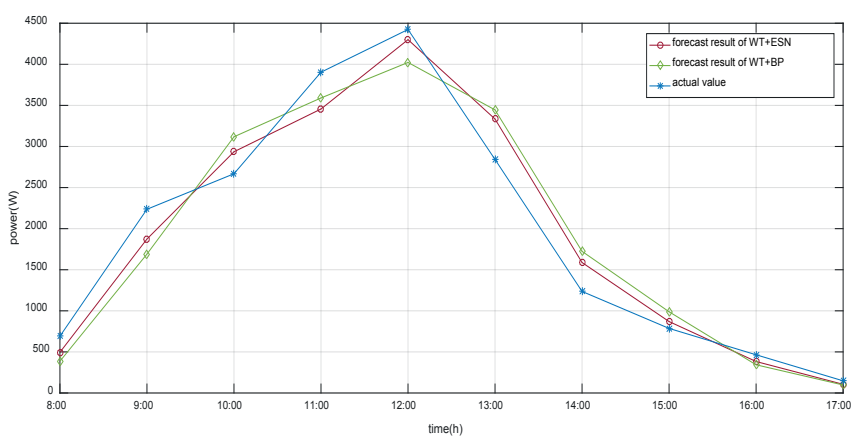

Fig. 11. WT $+B P$ and $W T+E S N$ model forecasted results (cloudy) 
The results graph show that the performance of the forecast model is improved obviously after the PV power sequence is de-noised by WT, and the forecast effect of the peak and the trough point of the power sequence is greatly improved, which shows that the wavelet transform prepares the PV sequence Is of great significance. ESN and BP neural network forecast curve comparison chart shows that, the average of the PV power forecasted by the ESN model is closer to the actual value and the forecast curve is more stable. But the power fluctuation forecasted by BP network is larger, which indicates that the forecasted performance of ESN is better than BP neural network, and the stability is stronger.

In order to evaluate the accuracy of the forecasting model, the relative error (RE), average percentage error (MAPE) and root mean square error (RMSE) are used to evaluate the forecast model effectively. The three kinds of errors are defined as Eq. (5), (6) and (7):

$$
\begin{gathered}
R E_{i}=\frac{y_{i}^{*}-y_{i}}{y_{i}^{*}} \times 100 \% \\
M A P E=\frac{1}{N} \sum_{i=1}^{N} \frac{\left|y_{i}^{*}-y_{i}\right|}{y_{i}^{*}} \times 100 \% \\
R M S E=\sqrt{\frac{1}{N} \sum_{i=1}^{N}\left(y_{i}^{*}-y_{i}\right)^{2}}
\end{gathered}
$$

In Eq. (5), (6) and (7):

$N$ - The total number of data sequences;

$i$ - The forecasted time value, $y_{i}^{*}$ is the actual value;

$y_{i}-$ The forecasted value.

The BP forecast model, the ESN forecast model, the $\mathrm{WT}+\mathrm{BP}$ forecast model and the WT+ESN forecast model were evaluated by using the above forecasted model evaluation index. Tab. 1 and 2 show the comparison of the forecast results in the sunny and cloudy days respectively. Fig. 12 and 13 show the relative error curves of the sunny and cloudy samples

\begin{tabular}{|c|c|c|c|c|c|c|c|c|}
\hline \multirow[b]{2}{*}{$\begin{array}{l}\text { Original } \\
\text { power } \\
\text { (W) }\end{array}$} & \multicolumn{2}{|c|}{ BP } & \multicolumn{2}{|c|}{ ESN } & \multicolumn{2}{|c|}{$\mathrm{WT}+\mathrm{BP}$} & \multicolumn{2}{|c|}{ WT+ESN } \\
\hline & $\begin{array}{l}\text { forecasted } \\
\text { power } \\
\text { (W) }\end{array}$ & $\operatorname{RE}(\%)$ & $\begin{array}{l}\text { forecasted } \\
\text { power } \\
\text { (W) }\end{array}$ & $\operatorname{RE}(\%)$ & $\begin{array}{c}\text { forecasted } \\
\text { power } \\
\text { (W) }\end{array}$ & $\operatorname{RE}(\%)$ & $\begin{array}{c}\text { forecasted } \\
\text { power } \\
\text { (W) }\end{array}$ & $\operatorname{RE}(\%)$ \\
\hline 6285 & 4624 & 26.43 & 7472 & -18.89 & 7188 & -14.36 & 7160 & -13.92 \\
\hline 9738 & 10852 & -11.44 & 10284 & -5.61 & 8269 & 15.09 & 8716 & 10.5 \\
\hline 12290 & 10524 & 14.37 & 11372 & 7.47 & 13449 & -9.43 & 13510 & -9.93 \\
\hline 13876 & 12569 & 9.42 & 11968 & 13.75 & 16274 & -17.28 & 14473 & -4.3 \\
\hline 14635 & 16401 & -12.07 & 15790 & -7.89 & 15984 & -9.22 & 15763 & -7.71 \\
\hline 14613 & 16752 & -14.64 & 16685 & -14.18 & 15979 & -9.35 & 15671 & -7.24 \\
\hline 13997 & 15309 & -9.37 & 15864 & -13.34 & 1563 & -11.67 & 14903 & -6.47 \\
\hline 12614 & 14307 & -13.42 & 12969 & -2.82 & 13872 & -9.97 & 13704 & -8.64 \\
\hline 10056 & 12989 & -29.17 & 12609 & -25.39 & 12559 & -24.89 & 11595 & -15.3 \\
\hline 5666 & 7702 & -35.94 & 7246 & -27.89 & 4083 & 27.94 & 4518 & 20.27 \\
\hline MAPE(\%) & \multicolumn{2}{|c|}{17.63} & \multicolumn{2}{|c|}{13.72} & \multicolumn{2}{|c|}{14.92} & \multicolumn{2}{|c|}{10.43} \\
\hline RMSE & \multicolumn{2}{|c|}{1840.16} & \multicolumn{2}{|c|}{1563.56} & \multicolumn{2}{|c|}{1636.29} & \multicolumn{2}{|c|}{1083.65} \\
\hline
\end{tabular}
respectively.

Tab. 1. A bolt sample four kinds of model forecast error value comparison

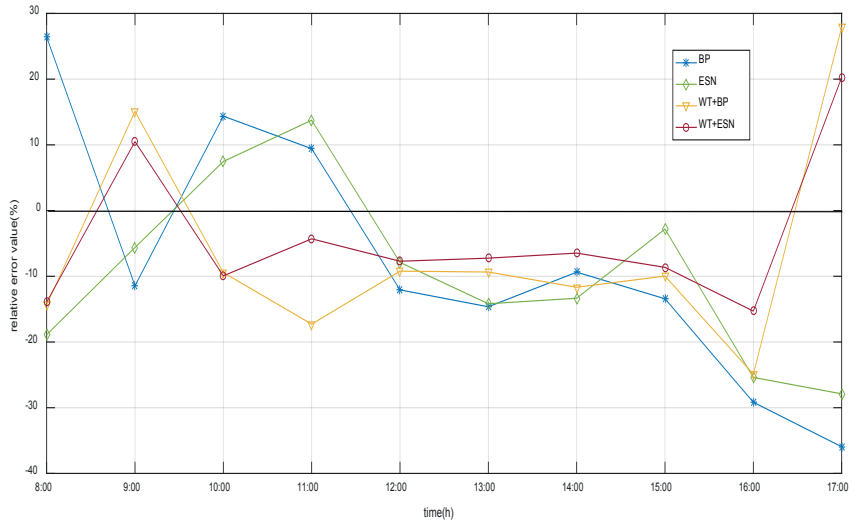

Fig. 12. Sunny samples relative error curve

Tab. 2. Cloudy sample four kinds of models to forecast the error value comparison

\begin{tabular}{|c|c|c|c|c|c|c|c|c|}
\hline \multirow{2}{*}{$\begin{array}{c}\text { Original } \\
\text { power (W) }\end{array}$} & \multicolumn{2}{|c|}{ BP } & \multicolumn{2}{c|}{ ESN } & \multicolumn{2}{c|}{ WT+BP } & \multicolumn{2}{c|}{ WT+ESN } \\
\cline { 2 - 9 } & power (W) & $R E(\%)$ & $\begin{array}{c}\text { Forecasted } \\
\text { power } \\
(\mathrm{W})\end{array}$ & $\mathrm{RE}(\%)$ & $\begin{array}{c}\text { Forecasted } \\
\text { power } \\
(\mathrm{W})\end{array}$ & $\mathrm{RE}(\%)$ & $\begin{array}{c}\text { Forecasted } \\
\text { power } \\
(\mathrm{W})\end{array}$ & $\mathrm{RE}(\%)$ \\
\hline 691 & 1006 & -45.64 & 442 & 36.06 & 387 & 44.05 & 496 & 28.31 \\
\hline 2236 & 1513 & 32.32 & 1470 & 34.27 & 1690 & 24.4 & 1874 & 16.21 \\
\hline 2668 & 3222 & -20.76 & 3058 & -14.63 & 3119 & -16.87 & 2936 & -10.03 \\
\hline 3904 & 5170 & -32.43 & 4309 & -10.37 & 3591 & 8.03 & 3456 & 11.47 \\
\hline 4424 & 5325 & -20.36 & 5095 & -15.17 & 4023 & 9.07 & 4300 & 2.8 \\
\hline 2842 & 3736 & -31.47 & 3479 & -22.42 & 3445 & -21.23 & 3334 & -17.31 \\
\hline 1236 & 1611 & -30.3 & 1670 & -35.13 & 1724 & -39.48 & 1588 & -28.46 \\
\hline 786 & 1020 & -29.81 & 940 & -19.59 & 988 & -25.76 & 870 & -10.62 \\
\hline 465 & 625 & -34.31 & 357 & 23.3 & 343 & 26.19 & 383 & 17.64 \\
\hline 148 & 111 & 25.16 & 118 & 20.36 & 97 & 34.16 & 105 & 28.8 \\
\hline MAPE(\%) & 29.36 & 23.03 & 24.92 & \multicolumn{2}{c|}{17.17} \\
\hline RMSE & 660.65 & 452.42 & 338.94 & 289.55 \\
\hline
\end{tabular}

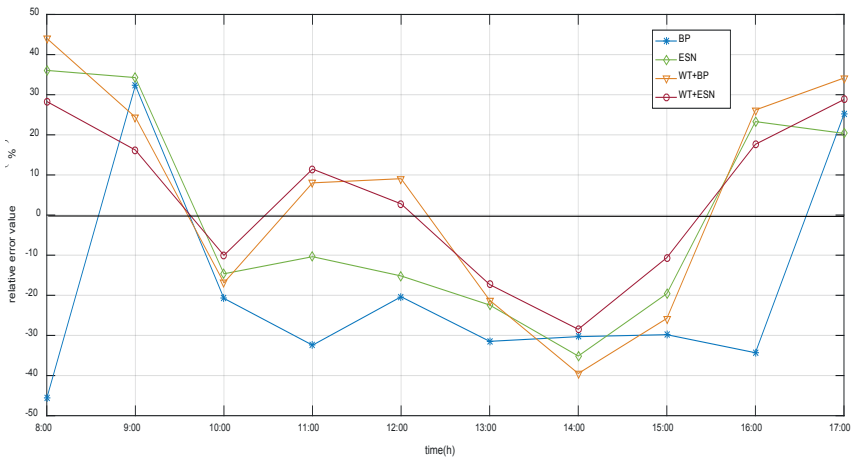

Fig. 13. Cloudy sample relative error curve

Tab. 1 and 2 show that the BP neural network is accurate for the forecasted results of sunny and cloudy days, but the errors at 8 o'clock and 17 o'clock are large. The main reason is that the morning and evening clouds are thick clouds, atmospheric transparency is not very high, the temperature difference is relatively large, low solar radiation, the impact of photovoltaic output, resulting in a larger forecast error. Because of the dynamic reserve pool, the ESN model has excellent nonlinear mapping ability and is less susceptible to local optimum and stronger stability. Therefore, the forecast accuracy of ESN is higher than that of BP neural network. After the de-noising of the PV power sequence by WT, the original power was decomposed into regular 
parts and random parts respectively, and the accuracy of the forecast model was improved. According to the error results of the four forecasted models, the forecast range of the WT+ESN model is smaller and more stable than the actual value. Regardless of which error index is used to evaluate, the forecast performance of WT+ESN is better than that of other three models, which proves the validity and accuracy of the designed model.

\section{CONCLUSIONS}

With the large-scale application of photovoltaic power generation technology and grid technology continues to mature, solar power generation has become an important part of the marine energy system. Based on the characteristics of the photovoltaic power generation system in the ship, this paper presented a high-precision power forecasting model for marine photovoltaic power generation system with antimarine environmental interference. Firstly, wavelet transform technology was used to preprocess the PV sequences in the marine environment to obtain the input PV components as the forecast model. And the ESN network structure and training process were built. The parameters and structure of the forecast model were determined by the Matlab platform and the collected PV data. Four forecast models of ESN, $\mathrm{BP}, \mathrm{WT}+\mathrm{ESN}$ and $\mathrm{WT}+\mathrm{BP}$ were established. The forecast performance of the model was evaluated by three kinds of error indexes RE, MAPE and RMSE. The results show that the forecast curve of WT+ESN model is smaller and more flat, and its overall forecast performance is better than the other three models.

\section{BIBLIOGRAPHY}

1. Li, J.: Solar Energy Application and Prospects in Marine Power Plant, Ship \& Ocean Engineering, Vol. 39, no. 4, pp. 70-72, 2010.

2. Guo, C., Sun, Y., Yuan, C., Yan, X.: Research on power load flow calculation for photovoltaic-ship power system based on PSAT in International Conference on Renewable Energy Research and Applications, 2015.

3. Klemchuk, P., Ezrin, M., Lavigne, G.: Investigation of the degradation and stabilization of EVA-based encapsulant in field-aged solar energy modules, Polymer Degradation \& Stability, Vol. 55,no.3,pp.347-365,1997.

4. Qiu, Y., Yuan, C., Sun, Y.: Review on the application and research progress of photovoltaics-ship power system, International Conference on Transportation Information and Safety, pp. 523-527, 2015.

5. Hirth, L.: Market value of solar power: Is photovoltaics costcompetitive, IET Renewable Power Generation, Vol. 39, no. 1, pp. 37-45, 2015.
6. Sun, Y., Yuan, C., Yan, X.: Theoretical model research on I-V characteristics of solar cell under the marine environment, International Conference on Transportation Information and Safety, pp. 877-882, 2015.

7. Kanemoto, T.: Dream of marine-topia: New technologies to utilize effectively renewable energies at offshore, Current Applied Physics, Vol. 10, no.2, pp. S4-S8, 2010.

8. Green, M.A., Emery, K., Hishikawa, Y.: Solar cell efficiency tables, Progress in Photovoltaics: Research and Applications, Vol.36, no. 18, pp. 346-352, 2010.

9. Li, W.C., Shi, Y.: Maximum power tracking photovoltaic power generation system and automatic tracking control research for ship, Ship Science and Technology,Vol.37,no .2, pp.136-139,2015.

10. Mahela, O.P., Ola, S.R.: Impact of grid disturbances on the output of grid connected solar photovoltaic system in IEEE Students' Conference on Electrical, Electronics and Computer Science, 2016.

11. Lou, X., Loparo, K.A.: Bearing fault diagnosis based on wavelet transform and fuzzy inference, Mechanical Systems \& Signal Processing, Vol. 18, no.5, pp. 1077-1095, 2004.

12. Lou, X., Loparo, K.K.: Bearing Fault Diagnosis Based on Wavelet Transform and Fuzzy Inference, Mechanical Systems and Signal Processing, Vol.18,no.5,pp. 1077-1095,2004.

13. Shi, Z.W., Min, H.: Ridge regression learning in ESN for chaotic time series prediction, Control \& Decision, Vol.22, no.3, pp. 258-257, 2007.

14. Malik Z K, Hussain A, Wu Q J.: Multilayered Echo State Machine: A Novel Architecture and Algorithm., IEEE Trans Cybern, Vol.47.no.4,pp. 946-959,2016.

15. Shi, G., Liu, D., Wei, Q.: Echo state network-based Q-learning method for optimalbattery control of offices combined with renewable energy, IET Control Theory \& Applications, Vol.11,no.7, pp. 915-922,2017.

16. Ji, L., Niu, D.X., Wu, H.M..: Daily Peak Load Forecasting Based on Bayesian Framework and Echo State Network, Power System Technology,Vol.36,no.11,pp. 82-86,2012.

17. Jaeger H.: Tutorial on Training Recurrent Neural Networks, Covering BPTT, RTRL, EKF, and the "Echo State Network" Approach in German National Research Center for Information Technology, 2002.

18. Wang S, Yang X J, Wei C J.: Harnessing Non-linearity by Sigmoid-wavelet Hybrid Echo State Networks (SWHESN) in Intelligent Control and Automation, 2006. 
19. Peng, Y., Wang, J.M., Peng, X.Y.: Researches on Time Series Prediction with Echo State Networks, Acta Electronica Sinica, Vol.38,no.b02,pp. 148-154,2010.

20. Luo, X., Li J., Sun Z.Q.: Review on echo state networks, Journal of University of Science and Technology Beijing Vol.34,no.2,pp. 217-222,2012.

\section{CONTACT WITH AUTHOR}

Du Xinhui

College of Electrical and Power Engineering Taiyuan University of Technology

Taiyuan, Shanxi, 030000

China 